\title{
German MODERN TALKING VS. IRANIAN MODERN TALKING. ZUR ANWENDBARKEIT DER KORPUS-ANALYSE als MitTel des Popmusikverstehens
}

\begin{abstract}
André Doehring
Isfahan (Iran)

Als sich der iranische Cousin an seine Zeit in Deutschland erinnert, kommt inm auch die Musik in den Sinn: »Modern Talking, ja! Das ist [Man beachte das Präsens!] schöne Musik, man bekommt gute Laune und kann dazu tanzen! Und Dieter Bohlen! Ein sehr guter Musiker, der ses - geschafft hat.« Mortezar ist nicht der erste, der mir in Iran begegnet und von den Vorzügen dieser deutschen Band berichtet. In Teheran legten Taxifahrer Modern Talking-Kassetten ein, sobald sich die Fahrgäste als Deutsche zu erkennen gaben, und dies bereits vor vielen Jahren, zu einer Zeit, als das Hören westlicher Popmusik im öffentlichen Raum noch empfindliche Strafen nach sich ziehen konnte. Heute ist dies ein wenig anders: Der Verwandte, der uns am nächsten Tag zu einer weiteren Einladung fährt, präsentiert uns bei geöffneten Autofenstern stolz eine Playlist sämtlicher Modern Talking-Hits: »Tolle Musik, nicht wahr!«
\end{abstract}

Gießen (Deutschland)

Fassungslosigkeit (»Das ist nicht dein Ernst!«), Besorgnis (»Bist du lebensmüde?«), bisweilen auch Häme (»Dann mal viel Vergnügen!«) kennzeichnen die Reaktionen von Kollegen und Freunden, wenn ich erzähle, dass ich an einer Korpus-Analyse aller Songs der ersten sechs Modern Talking-Alben arbeite. Diese exemplarisch zitierten Aussagen entstammen einem Arbeitsjournal, das ich während der Arbeit führte und das außerdem manche Selbstbeobachtung enthält: etwa die zu Beginn der Arbeit auffällig lange Phase der Überwindung meines Widerwillens, sich analytisch mit dieser Musik auseinanderzusetzen oder meine anschließende eigentümliche Faszination für den Gegenstand, die zwischen Freude - auch des Wiederent- 
deckens - und Unbehagen pendelt. Scham ist diesen Aufzeichnungen ebenfalls zu entnehmen, in einem Fall ausgelöst durch eine im Bekanntenkreis kursierende heimlich gefilmte Sequenz, die mich zeigt, wie ich meinen Körper zum in hoher Lautstärke spielenden »You're My Heart, You're My Soul « (Modern Talking 1984) vor- und zurückbewege. Dass meine Beteuerung, ich zählte nur die Takte aus, das Gelächter der Anderen nur verstärkte, ja dass ich überhaupt glaubte, diese Entschuldigung anbringen zu müssen - dies alles deutet darauf hin, dass etwas im Vergleich zur iranischen Rezeption grundsätzlich Anderes im Gange ist, wenn ein deutscher Musikwissenschaftler eine der (zumindest nach Plattenverkäufen gemessen) erfolgreichsten bundesdeutschen Bands der Popmusik zum Gegenstand wählt.

Wie diese einleitenden Beispiele zeigen, spielen in der Beschäftigung mit Musik von Modern Talking - und selbstverständlich nicht nur dort, sondern stets - kulturelle, soziale sowie insbesondere disziplinäre und methodische Aspekte eine große Rolle. Auf der Basis derselben Klänge haben sich eine iranische und deutsche Rezeption entwickelt, deren einzige Gemeinsamkeit in der lange bestehenden Unterdrückung des Gegenstands zu liegen scheint. Für die bundesdeutsche Musikwissenschaft gab es natürlich keine offiziellen Verbote wie in der Islamischen Republik Iran, doch wie gezeigt werden wird, war die Popmusik von Bands wie Modern Talking lange außerhalb der musikologischen Grenzen angesiedelt. Dies ist ebenso zu bedauern wie zu ändern, denn es sind gerade die Grenzübertritte, die einen anderen Blick auf das soeben verlassene Eigene ermöglichen. Im Folgenden wird mit einer Korpus-Analyse ein erster Blick auf die klangliche Struktur der Musik von Modern Talking geworfen. Allerdings, so viel sei im Voraus gewarnt, verraten uns die Analyseergebnisse aufgrund der Spezifität der Methode mehr über die deutsche musikwissenschaftliche Rezeption als über die iranische. Daher ist die grundlegende Fragestellung dieser Abhandlung, was ich oder allgemeiner: was ein/e Musikwissenschaftler/in verstehen kann, wenn man sich dem vielen Menschen bestens bekannten >Fremden< zuwendet. Ihrer zumindest einstweiligen - Beantwortung am Ende gehen Überlegungen zur ideologischen Grenzziehung der Musikwissenschaft, zur Analyse als Methode sowie die Ergebnisse und die Interpretation der Korpus-Analyse voraus. 


\section{Über die Grenzen der musikwissenschaftlichen Wahrnehmung - hinaus!}

Unlängst machte der US-amerikanische Musikhistoriker Elijah Wald in den Beiträgen zur Popularmusikforschung (2014) darauf aufmerksam, dass die Popmusikforschung interessanterweise gerade diejenigen Musiken aus ihrem Forschungsbereich ausschließt, die in ihren jeweiligen historischen Kontexten besonders populär waren. In der Tat: An Popmusik interessierte Musikwissenschaftler untersuchen äußerst selten die Hits der Charts, der Volksfeste oder der ländlichen Diskotheken. ${ }^{1}$ Dies liegt, wenn wir Walds Argumentation folgen, in der Tatsache begründet, dass die Geschichte der populären Musik zwar immer auch eine Geschichte des Tanzens zu dieser Musik war und ist, letztere allerdings in der Popmusikhistoriographie keinen Niederschlag gefunden habe. Den rhetorisch zugespitzt genderstereotypen, allerdings angesichts der realen Verteilung der Geschlechter in den höheren Positionen der Wissenschaft wie auch des Musikjournalismus nicht falschen Grund dafür benennt er folgendermaßen: ${ }^{2}$

»There is typically a huge divide between people who care about preserving and analyzing music and people who just like to dance. I often describe that divide in a way that is kind of a joke, but also uncomfortably true: The typical consumer of Western popular music is a teenage girl who likes to dance, while the typical critic or scholar is a man who had no dates in high school« (Wald 2014: 29).

Freilich tappen nun viele musikwissenschaftliche Leser - wie auch der Autor - in die Falle und werden ihre natürlich umfangreichen jugendlichen Liebes- wie Tanzerfahrungen zum Gegenbeweis anführen wollen. Trotzdem kommt man nicht umhin, Walds Diagnose des herrschenden Trends in der Popmusikforschung zuzustimmen: »| continue to see a lot of academic attention being paid to music favored by men who don't dance - for example,

1 Ausnahmen bestätigen die Regel; vgl. Thomas Phleps' (2014) Analysen bundesdeutscher Schlagertümlichkeit anhand ihres »erfolgreichsten Duos«, den Amigos.

2 In Nordrhein-Westfalen sind im Jahr 2013 22,5\% (W2/C2/C3) bzw. 17,5\% (W3/ C4) der Professuren an allen Hochschulen weiblich besetzt (vgl. http://www. genderreport-hochschulen.nrw.de/no_cache/statistikportal; Zugriff 23.1.2015). Im Musikjournalismus ist im Jahr 2008 die Geschlechterverteilung in den Redaktionen noch geringer (bloß ein Neuntel ist weiblich), unter den freien Mitarbeitern sind Frauen höchstens zu 17,7\% (Intro), wenigstens zu 8,6\% (Spex) vertreten (vgl. Doehring 2011: 168). 
punk, and rap, and heavy metal - while a lot of other, often more popular music is ignored « (ebd.: 34). ${ }^{3}$

Ein derartig weitreichender Ausschluss von Musiken aus dem Repertoire einer Disziplin ist, den Erkenntnissen der Critical bzw. New Musicology folgend, stets mit der Ausübung von Herrschaft verbunden. Die andere Musik ist eben immer die Musik der Anderen, denen so die Zugänge zum Feld sowie die Ansprüche auf Herrschaft darin verwehrt werden. Im Hinblick auf die anglophon dominierte Popmusikforschung muss der/die/das Andere, üblicherweise durch die Trias Race - Class - Gender definiert, um die Kategorie des Ortes erweitert werden, da hier nicht-englischsprachige Musik sowie nicht dem United Kingdom oder den USA entstammende MusikerInnen wenig Beachtung finden. Selbst die deutschsprachige Popmusikforschung scheint dieser - unbewusst verlaufenden - Legitimitätsprüfung des musikalischen Gegenstands zu erliegen, wie Wald (ebd.: 29f.) mit dem privilegierten Blick des Außenstehenden feststellt: Als einen blinden Fleck der deutschsprachigen Popmusikforschung benennt er bspw. die ausgebliebene Beschäftigung mit Boney M., die mit überwiegend weiblichen und ausschließlich farbigen Mitgliedern vom Produzenten Frank Farian (geb. Franz Reuther) in Offenbach-Bieber »selbst erfundene « (Eidam/Schröder 2001: 15) und - wiederum bezogen auf die Plattenverkäufe - populärste Formation aus >diesem unseren Lande. Dass außerdem diese öffentlichen Boney M.Performer nicht an der Komposition und nur zum Teil an der Produktion der funktional auf einen Disco-Kontext zielenden Songs beteiligt waren, mag als weiteres Indiz für die in der Popmusikforschung im Stillen herrschenden Prämissen an ihre Gegenstände dienen.

Die Gründe für diese besonderen Grenzziehungen liegen gewiss in der langen Geschichte der Mutterdisziplin Musikwissenschaft als Kunstwissenschaft, in der die Werke ${ }^{4}$ der Wenigen, indes Mächtigen bzw. durch diese Protegierten, nicht jedoch die Musiken der Vielen kanonisiert wurden. Auch wenn sich vielerorts dieser Anspruch hin zu einer kulturwissenschaftlichen Orientierung gewandelt haben mag (vgl. bspw. die Beiträge in Calella/Urbanek 2013), ist doch immer noch eine disziplinäre Faszination für diejenige abseits des Mainstreams klingende populäre Musik zu beobachten, der eine gewisse handwerkliche Brillanz und kunstvolle Gestaltung durch ein sgenia-

3 Auch wenn etwa im Heavy Metal unter gewissen Umständen getanzt wird (zumindest im selben Maße, wie dort gesungen wird), bleibt Walds Befund prägnant.

4 In dem ohnehin problematischen Begriff des Werks, vom individuellen >Genie des Komponisten geschaffen, wird eine weitere Grenzziehung in dem Moment ersichtlich, wo er sich der problemlosen Anwendung auf die Musik von Modern Talking widersetzt. 
les Individuum (seltener ein Duo, fast nie ein Kollektiv) zuerkannt werden kann. Und dies selbstverständlich nur in den Fällen, in denen Musikwissenschaft mit dem ihr zur Verfügung stehenden und an einem Kanon von Werken etablierten Begriffs- und Wahrnehmungsapparat erkennen kann, wie das musikalische und instrumentale Handwerk ausgeübt wird. Synthetisch resp. digital erzeugte Musik wie die von Modern Talking stellt insofern immer noch einen relativ neuen Gegenstand dar (vgl. zur Lage der elektronischen Tanzmusik im Fach Doehring 2015: 134f.).

Vor diesem Hintergrund ist es verständlich, warum bspw. die anglophone Musikwissenschaft einen eklatant größeren Literaturkorpus über die Musik der Beatles bilden konnte, die Musik von Modern Talking bisher aber zu so wenig Resonanz in der deutschsprachigen Musikwissenschaft führte bzw., um Karl Valentin zu paraphrasieren, warum sie noch nicht einmal durch die Disziplin ignoriert wurde: Modern Talkings Musik war und ist populär, sie ist kommerziell erfolgreich, zu ihr wurde und wird getanzt dies alles gilt freilich auch für die Beatles (wobei deren späten Alben, die im Mittelpunkt der musikwissenschaftlichen Rezeption stehen, kaum die Eignung zur Tanz-, sondern zur Kunstmusik attestiert wird). Aber entscheidend ist, dass Modern Talkings Musik aus Deutschland kommt, dass sie mit einem anderen als dem klassischen (Rock-)Instrumentarium erzeugt wird und dass ihr keine kunstvolle Gestaltung attestiert werden kann bzw. darf. Kurz: Sie findet jenseits der musikologischen Wahrnehmung statt.

Dieser Ausschluss stellt zum einen natürlich den herrschenden Anspruch an das eigene Arbeitsfeld der hier sog. populären Musik infrage. Zum anderen belegt das derart bezeugte Desinteresse an den Klängen sowie an den Kontexten ihrer Produktion, Distribution und Rezeption einen Rückzug der Disziplin - oder, falls man denn je wirklich ausgezogen war: ihr Einigeln in den sprichwörtlichen Elfenbeinturm weitab einer popmusikalischen Realität unserer Kultur, zu der man nur bei gutem Wetter und richtig stehendem Wind die Fenster ein wenig zu öffnen pflegt. Ob wir es mögen oder nicht: Will die popmusikforschende Musikwissenschaft als Kulturwissenschaft agieren, muss sie diese nach wie vor bestehenden Grenzen zum Populären überwinden - sei es als Fan (ein für diesen Bereich zwar seltener, ansonsten durchaus legitimer Zugang, wie wir durch die o.g. Rap-, Punk- und Heavy Metal-Forscher wissen) oder, wie im vorliegenden Fall, als Pflichtverteidiger. 


\section{Ergebnisse der Korpus-Analyse}

Popmusikforschung ist ein notwendigerweise, d.h. dem Gegenstand adäquates interdisziplinäres Unternehmen. Indes hat sie sich aufgrund ihrer besonderen Geschichte (vgl. Rösing 2002; Pfleiderer/Grosch/Appen 2014) mit ihrer starken sozialwissenschaftlichen Verwurzelung nur selten um die Klangstruktur gekümmert. Das ist selbstverständlich den Sozialwissenschaften aufgrund dort fehlender Methoden nicht vorzuwerfen. Trotzdem ist das derart in die Popmusikforschung eingebrachte mangelnde Bewusstsein für den gesamten Gegenstand sicher nicht hilfreich, wird somit doch suggeriert, dass die klangliche Struktur vernachlässigt, möglicherweise gar - dem Anschein nach folgenlos - ignoriert werden könne. Musikwissenschaft kann mithilfe der Methode der Analyse als einzige der an der Erforschung populärer Musik beteiligten Disziplinen zur Klanggestalt fundierte Aussagen treffen (Appen/Doehring 2014: 219); sie besitzt, um werberisch eine Lanze zu brechen, die USP (unique selling proposition) im Feld, das - zwar doppelt gemoppelt dummdeutsche, dessen ungeachtet weiterhin betriebswirtschaftlich so genannte - Alleinstellungsmerkmal.

In Anbetracht der Forschungslage zur Musik Modern Talkings erscheint es mir angeraten, einen ersten analytischen Versuch mithilfe einer Analyse zu unternehmen, die nicht den Einzelfall, sondern das Verbindende in allen Veröffentlichungen von Modern Talking sucht. Notwendigerweise rückt somit die individuelle Struktur und Rezeption eines Songs in den Hintergrund des Interesses, dafür treten jedoch Gemeinsamkeiten der musikalischen Struktur aller Songs hervor, die in der Einzelanalyse nicht beobachtet werden können. Als Grundlage dient mir der Korpus von 59 Songs, die auf den ersten sechs Studioalben zwischen April 1985 und November 1987 erschienen sind. Die Beschränkung auf diese erste Phase von Modern Talking erscheint insofern berechtigt, als hier die Grundlage der musikalischen Betätigung auch der zweiten Phase um die Jahrtausendwende gelegt wurde. ${ }^{5}$ Hinsichtlich der untersuchten Parameter musste aus arbeitsökonomischen Gründen eine Reduzierung vorgenommen werden: Alle Songs wurden bezüg-

5 Modern Talking waren von 1984-1987 und 1998-2003 aktiv. Die erste Phase ist prägend für die Musik und ihre Rezeption, da hier der seitdem für die deutschen Charts geltende Rekord von fünf konsekutiven Nr.1-Singles einer Band aufgestellt wurde. Außerdem besteht das nur hinsichtlich des immensen kommerziellen Erfolgs nicht falsch betitelte Comeback-Album Back For Good (1998) zu fast 80 Prozent (14 von 18 Songs) aus in erster Linie soundtechnisch überarbeiteten Versionen von Songs aus der ersten Phase. 
lich ihrer formalen, harmonischen und rhythmischen Strukturen sowie ihrer Instrumentation und ihres Tempos analysiert.

Tempo ist einer der wesentlichen Parameter für die Etablierung von musikalischen Ähnlichkeitsbeziehungen, wie der Chef des Pandora Music Genome Project Steve Hogan aus der eigenen Arbeit berichtet (vgl. Byron 2014). ${ }^{6}$ Dem bereits vor Modern Talking jahrelang als Auftragsproduzent für Intersong/Warner Chappell, dann für Hansa Musikproduktion tätigen Dieter Bohlen, der sämtliche Songs für Modern Talking komponierte, muss diese Bedeutung des Tempos klar gewesen sein: Die 59 Songs haben ein durchschnittliches Tempo von 108 bpm bei einer Standardabweichung von 16,3. Wie aus Abb. 1 ersichtlich wird, weichen elf Songs deutlich von diesem Durchschnitt ab (übrigens auch thematisch, denn dies sind die Weihnachtsund Liebes-Balladen).

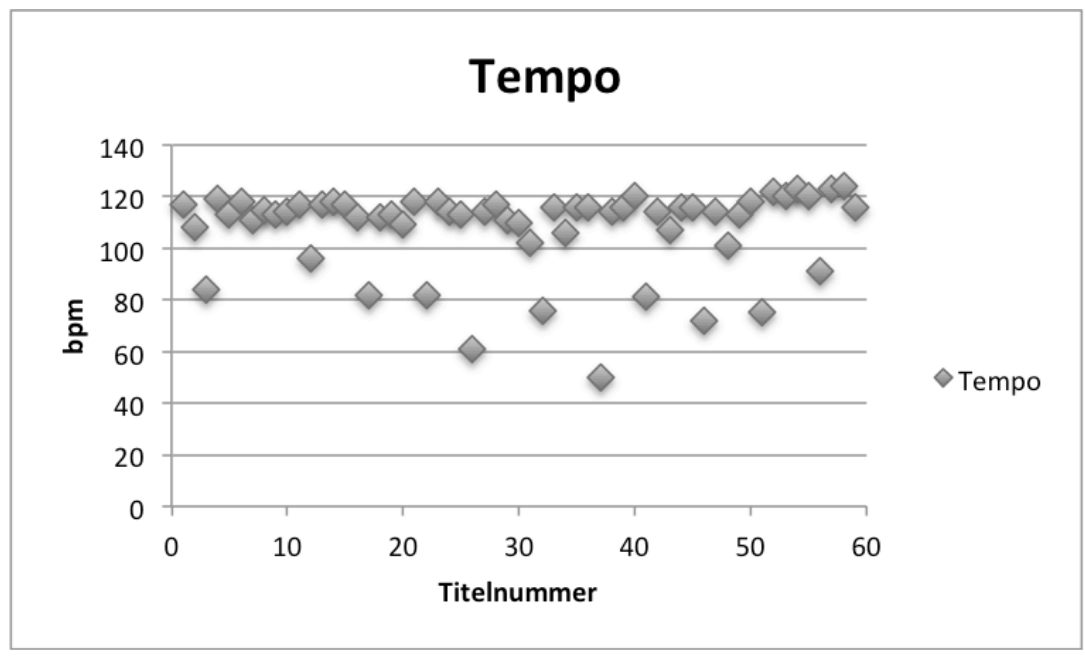

Abbildung 1: Tempo (in bpm) der Modern Talking-Songs

Rechnet man diese langsameren Titel heraus, ergibt sich für die verbleibenden 48 Songs das nun etwas schnellere Durchschnittstempo von 115 bpm, die Standardabweichung ist auf 4,9 gesunken. $\mathrm{Zu}$ dieser überraschenden Einheitlichkeit im Tempo tritt eine weitere hinzu: Die elf langsameren Songs sind auf jedem der sechs Alben - die bis auf das erste Album durchweg zehn Songs enthalten - an denselben Stellen der Ablaufprogrammierung zu finden, nämlich immer auf Platz 2 oder 3 sowie auf Platz 7 bzw. 8. Für die >Marke<Modern Talking hat das zur Folge, dass ihre Platten als qualitativ

6 Dort sind - tatsächlich! - Musikwissenschaftler für die analytisch begründete Kategorisierung von Popmusik verantwortlich, deren Ergebnisse dann einen möglichst homogenen Musikfluss auf dem Internetradiosender pandora.com ermöglichen sollen. Insgesamt werden für die Analyse ca. 450 Kategorien benutzt (vgl. http://www.pandora.com/about/mgp; Zugriff 1.2.2015). 
ähnlich rezipiert werden können: Man kann zu egal welchem dieser Alben durchtanzen, was bei einem relativ homogenen und gemäßigt schnellen Tempo von 115 bpm ohne Probleme möglich ist - allerdings nur, wenn man daran denkt, an den immer gleichen Stellen der Alben einen Song zu überspringen.

Ein ähnlich hoher Grad an Ähnlichkeit ist in formaler Hinsicht zu entdecken. Die typische Modern Talking-Songform ist: Intro - Verse - ChorusSektion - Re-Intro - Verse - Chorus-Sektion - Chorus-Wiederholungen. Jeder Formteil besteht aus meist zwei Wiederholungen achttaktiger Glieder; auftretende Abweichungen in der Länge (meist in Form eines zusätzlichen Takts) liegen in ihrer Funktion begründet, den folgenden Formteil anzukündigen (»episodic marker « nach Tagg 1992: 377). Andere in der Popmusik geläufige Songformen (bspw. AABA, AAA, Blues-Form, Track-Form etc.) sind im Korpus nicht zu entdecken. Eine Bridge (vgl. zur Terminologie und Geschichte Appen/Frei-Hauenschild 2015) als variierendes Element dieser strengen Verse-Chorus-Form taucht in vier Songs $(6,8 \%)$ auf. In knapp $12 \%$ der Fälle (sieben Songs) eröffnet der Song nach dem Intro mit dem Chorus, eine in der Popmusik durchaus (ein-)gängige, hier aber überraschend wirkende Formvariation. ${ }^{7}$ Diese bemerkenswerte Einheitlichkeit der Form ist auch in der konkreten Ausgestaltung der Chorus-Sektion erkennbar. In gut zwei Dritteln $(67,8 \%)$ des Korpus besteht dieser Formteil aus einer dreiteiligen Anlage von Pre-Chorus, Chorus und dem chorisch im Kopfstimmregister gesungenen Post-Chorus. ${ }^{8}$ In $81,4 \%$ aller Songs sind ein Chorus und ein PostChorus vertreten, in bloß fünf Fällen $(8,5 \%)$ gibt es einen einteiligen Chorus. Bohlen weiß um die Bedeutung dieses Formteils für die Erinnerbarkeit und somit potentiell höhere Verkäuflichkeit seiner Musik, daher bietet er den Hörern in den Chorus-Teilen viele hooks an, die vorbereitend und öffnend (Pre-Chorus), hochgradig eingängig und sanglich (Chorus) sowie auf einem höheren Energielevel bestätigend und weiterführend (Post-Chorus) funktionieren. Insbesondere der Post-Chorus signalisiert in seiner Zitation des durch die Bee Gees verbreiteten chorischen Kopfstimmregistergesangs die Funktion als Tanzmusik im Disco-Kontext. Der Post-Chorus ist im Ver-

7 Diese für den vorliegenden Korpus so zu nennenden >Überraschungen< im formalen Ablauf beruhen natürlich auf der ansonsten strikten Durchführung der benannten Modern Talking-Standardsongform.

8 Am Beispiel der ersten Nr. 1-Single »You're My Heart You're My Soul« illustriert: Pre-Chorus ( $\gg$ I'm dying in emotion / In my world of fantasies / Living in my, living in my dreams «) - Chorus (»You're my heart...«, gesungen von Thomas Anders im Bruststimmregister) - Post-Chorus (»You're my heart...", gesungen im Kopfstimmregister, in diesem Fall von Dieter Bohlen; vgl. die Ausführungen zum Produktionsteam auf S. 131). 
gleich zum vorhergehenden Chorus anders textiert, oft folgt er auch einer neuen harmonischen Struktur; hier wurde kompositorisch wie auch produktionstechnisch mit den mehrfach übereinandergeschichteten Chören viel Arbeit investiert. Da er in vier Fünfteln aller Fälle vorhanden ist, kann man ihn als zentralen Bestandteil des typischen Modern Talking-Sounds benennen.

Auch in harmonischer Hinsicht ist die Ähnlichkeit des Materials evident. Mehr als die Hälfte der Songs (52,6\%) stehen in Moll, ${ }^{9}$ gerade einmal 15,2\% in Dur. Das fehlende knappe Drittel des Korpus (32,2\%) variiert das harmonische Geschlecht, in der Regel für den Chorus. Diese Modulationen gehen zu fast zwei Dritteln (63,2\%) von einem in Moll stehenden Verse in eine in Dur stehende Chorus-Sektion, das folgende Re-Intro steht wieder in Moll. ${ }^{10}$ Fast sämtliche Modulationen wechseln in die Paralleltonart (94,7\%).

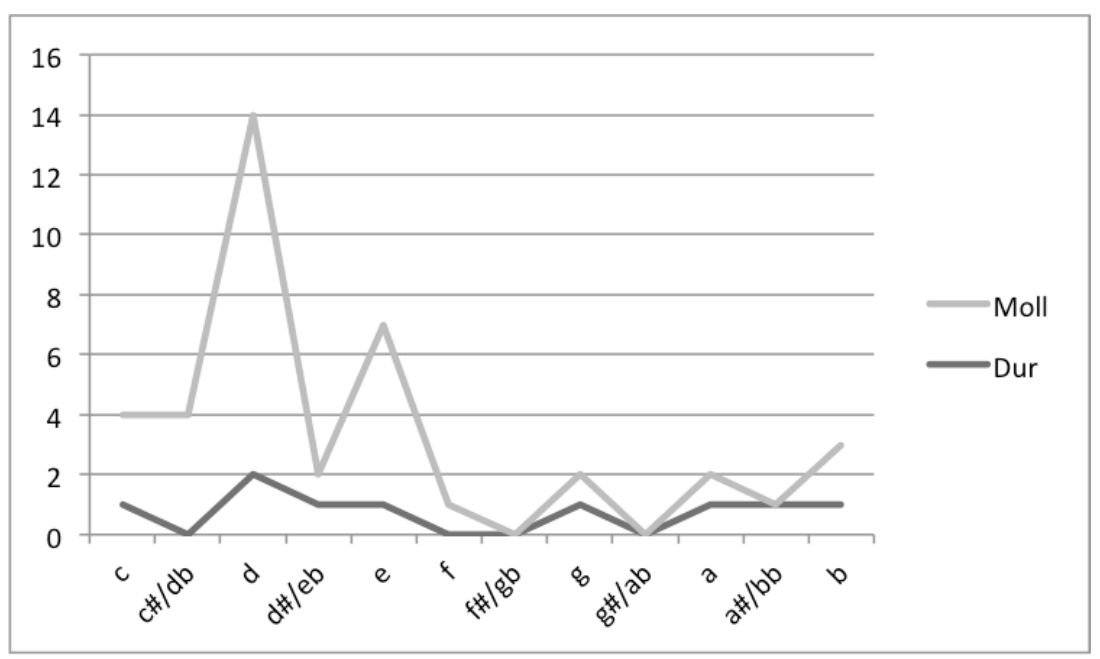

Abbildung 2: Tonart und Geschlecht der Modern Talking-Songs

Die favorisierte Tonart ist d-Moll (s. Abb. 2), was möglicherweise mit der Orientierung am Ambitus von Lead- wie Backing-Gesang, vielleicht aber auch mit der Produktionspraxis begründet werden kann: Bohlen produzierte die Demos in seinem Kellerstudio auf einem Sequential Circuits Prophet-5 Synthesizer; ${ }^{11}$ als Gitarrist mag der Griff in die Tonarten mit wenigen Vorzeichen eine Rolle gespielt haben. Diese instrumentaltechnische Erklärung

9 Dieter Bohlen macht dafür schlicht die »russische Seele« (Bohlen 2002: 12) seiner Großmutter verantwortlich: »Dieses ganze Moll-Feeling in meiner Musik habe ich von ihr« (ebd.).

10 Somit ist der Eindruck der vorherrschenden Mollharmonik beim Hören eines Albums noch höher, da dieses modulierende Drittel aller Songs in Moll beginnt.

11 Zum Kauf des Synthesizers sei er durch Phil Collins' Hit »In The Air Tonight« (1981) angeregt worden, auf dem er nun fortan jeden seiner Hits produzierte bei Bohlen läuft er seitdem unter dem »Code-Name[n] Profit $\mathrm{V}_{«}(\mathrm{vgl}$. Bohlen 2002: 49f.) 
kann auch ursächlich für das seltene Vorkommen von Akkord-Alterationen sein, die erst auf dem sechsten Album in größerem Ausmaß zu hören sind. Ihr äußerst seltenes Erklingen auf den Alben zuvor wird aber sicherlich auch auf ästhetischen Maßgaben beruhen, die dann die Produktivkräfte im Studio umzusetzen hatten (s.u.).

Die Songs in einer Dur-Umgebung bedienen sich ausschließlich einer einfachen Funktionsharmonik. Die Songs in Moll unterliegen einer modalen Harmonik: Auf den ersten drei Alben ist nur der äolische Modus ( $i-i v-v)$ präsent, ab dem vierten Album sind harmonisches und melodisches Moll oder der dorische Modus als Alternativen zum immer noch vorherrschenden natürlichen Moll zu entdecken. Bohlen vermeidet jegliche harmonische Ambiguitäten: Intro, Pre- oder Post-Chorus enden oft auf einer öffnenden $V$ in einer Dur- oder auf einer bVII in einer Moll-Umgebung, die ihm in der äolischen Moll-Umgebung geeigneter als die v erscheint. Diese harmonischen episodic marker werden in ihrer Funktion mitunter durch den erwähnten zusätzlichen Takt und/oder durch eine rhythmische Figur (auf der $\vee$ folgen nach einem Volltakt Viertelnoten fünf Achtel) unterstützt, die aus dem Schlager bekannt sind.

Als typische Kadenz im Korpus ist die in einer Mollumgebung zu findende iv - bVII $(-i)$ zu nennen, die oft für den Wechsel in die Durparalleltonart genutzt werden kann (ii $-\mathrm{V} \rightarrow \mathrm{I}$ ). Des Weiteren sind in vielen Modern Talking-Songs Akkord-Shuttles zwischen $\mathrm{i}-\mathrm{v}$ oder $\mathrm{i}-\mathrm{iv}$ zu hören. Interessanterweise wechseln sie sich oft innerhalb eines Songs ab: Folgt bspw. der Chorus einer $\mathrm{i}-\mathrm{v}$-Verbindung, wechselt der anschließende Post-Chorus zwischen i - iv (»Let's Talk About Love«, Modern Talking 1985d; »Save Me Don't Break Me«, Modern Talking 1986c). Obwohl Bohlen nur äußerst selten die sechs Akkorde des zugrunde liegenden harmonischen Rahmens verlässt, ist er doch bestrebt, stereotype harmonische Verbindungen außer den erwähnten zu umgehen. Besonders auf den letzten Alben ist das Bemühen um eine Klischeevermeidung erkennbar: Hier wirken manche harmonische Verbindungen tatsächlich süberraschend<, da ein kadenzieller Sinn nicht mehr offensichtlich ist.

Die Instrumentation der Songs weist ebenfalls eine hohe Ähnlichkeit auf. Alle Instrumente stammen, bis auf den Gesang sowie Rhythmus- und LeadGitarren, aus dem Synthesizer. Insbesondere der Synthie-Bass, der sich zumeist auf pentatonische Pattern beschränkt, und der Drum-Computer mit der grundlegenden Four-to-the-Floor-Bassdrum lassen auch auf der Soundebene den Versuch erkennen, an zeitgenössische Disco-Kontexte anzuschließen. ${ }^{12}$

12 Diskotheken hatten sich allerdings, auch das ist zu konstatieren, in New York, Chicago, London oder Ibiza, das Mitte der 1980er als Geheimtipp galt, anderer 


\section{Interpretation}

Analyse ist ein Mittel der Popmusikforschung zum Verstehen. Sie ist somit kein Zweck, erst recht kein Selbst-Zweck. Unnachahmlich fragt Ekkehard Jost (1999a: 17) in der Jazzforschung nach dem Sinn von Analyse: »Alles transkribiert, alles analysiert, alles unter Kontrolle. Was nun? « Allan Moores (2009: 412) Version der Frage ist genauso kurz: "So what?« Beide stellen die bloße parametrische Zergliederung von Klang als sinnlos infrage, wenn sie nicht anschließend die Grundlage einer Interpretation durch das analysierende Subjekt hergibt. Doch was wollen bzw. was können wir verstehen?

Ein typisches und weit verbreitetes Ziel ist das Verstehen des musikalischen Funktionierens populärer Musik, das Jost (1999b: 103) in Bezug auf Jazz als »Verstehen, wie Jazz gemacht wird « bezeichnet. Analyse ist dann bspw. ein Mittel zum pädagogischen Zweck der Reproduktion, der wissenschaftlichen Demonstration oder Aufklärung bis hin zum Beleg für den Kunstcharakter eines Musikstücks. Letzteres war lange Zeit alleiniger Zweck von Analyse. Eine Folge des subjective turn (vgl. Doehring 2012; Moore 2012) ist aber, dass man das bei manchen Hörern - und vielen Musikwissenschaftlern - verbreitete Verständnis von populärer Musik als Kunst als einen möglichen Ausgangs- wie Endpunkt der Analyse bestimmen kann. Ein anderes Ziel entspricht der grundlegenden Aufgabe systematischer Musikwissenschaft, wie Helga de la Motte-Haber (1982: 12) sie benennt, nämlich der des »Verstehens des Musikverstehens ${ }^{13}$. Auch wenn derzeit im subjective turn die eigene Rezeption der Analysierenden im Mittelpunkt steht, sind prinzi-

Musik als der von Modern Talking zugewandt - House, ersten Techno-Tracks und EBM. Man darf annehmen, dass weder Bohlen noch die Labelbetreiber in enger Beziehung zu diesen Orten standen, sondern ihre Konstruktion von Disco darauf errichteten, was in Hamburg, Sitz des Labels und Bohlens Wohnort, zu diesem Zeitpunkt aus der Discowelle Ende der 1970er Jahre nachhallte. Auch auf der Formatebene ist Disco als Kontext erkennbar, ohne dass er sich in dem Produkt niederschlägt: Alle Singles sind auch als 12"-Single veröffentlicht worden (Modern Talking 1984, 1985a, 1985c, 1986a, 1986b, 1986d, 1986f, 1987a, 1987c). Nur die letzte überwindet die Grenze von sechs Minuten, die anderen als »Special-Dance-« oder »Long-Version « angepriesenen Aufnahmen sind gerade einmal fünf Minuten lang. Es darf vermutet werden, dass hier nicht ein kleiner DJ-Markt, sondern die Zielgruppe der Fans im Visier der Plattenfirma stand, um mit dieser minimalen Produktdiversifizierung den Absatz zu erhöhen.

13 La Motte-Haber bezieht sich auf das von Guido Adler stammende " Reciprocitätsverhältnis zwischen Musik und Hörer «. Sie ist sich außerdem bewusst: »Die Beschäftigung mit den Schwierigkeiten, Bedingungen, Möglichkeiten und Auswirkungen des Musikverstehens bedarf musikgeschichtlichen Wissens vor allem in Form von Kultur- und Rezeptionsgeschichte (ebd.). 
piell alle musikalischen Praxen (Blaukopf 1984) von Musik - auch diejenigen Dritter - mögliche Gegenstände der Analyse. Ein sozialgeschichtlich interessiertes Verstehen, das den Wandel im Verständnis und auch in der Funktion eines Musikstücks anstrebt (Jost 1999b: 99), profitiert von der analytischen Methode, da diese die klanglich relevanten Parameter herausarbeitet, die Gegenstand der musikalischen Verfahren auf Musikerseite sind oder diejenigen, auf die sich eine zeitgenössische Rezeption bezieht.

Das in meinen Augen Typische aller Modern Talking-Songs ist das auf vielen Ebenen aufgezeigte hohe Maß an Ähnlichkeit. Diese Standardisierung des musikalischen Materials kann uns somit erstens Aufschluss bieten über die Umstände der Produktion von populärer Tanzmusik in der BRD in den 1980er Jahren. Zweitens bildet diese klangstrukturelle Ähnlichkeit die Basis meiner Rezeption, die im Anschluss auf ihre Spezifität hin untersucht wird. Doch zunächst zum Kontext der Produktion.

Wenn wir uns die Daten und Anzahl der Veröffentlichungen vor Augen führen und zudem die Berichte von Dieter Bohlen und Thomas Anders in ihren Autobiografien (Bohlen 2002; Anders 2011) über die enormen Promotion- und Touraktivitäten im Umfeld dieser Veröffentlichungen heranziehen, wird deutlich, dass Bohlen die für die Entwicklung einer musikalischen Vielfalt notwendige Zeit gefehlt hat, wie sie etwa die Beatles nach der Beendigung ihrer Liveauftritte ab 1966 hatten. Es ist allerdings fraglich, ob er diesen Freiraum tatsächlich vermisst hat: ${ }^{14}$ Denn Bohlen ist zum Zeitpunkt des Erscheinens der ersten Single bereits ein seit Jahren in der Musikindustrie beschäftigter Musikproduzent, erst für Intersong/Warner Chappell, ab 1980 für die Hansa-Musikproduktion. Er hat also die in der Musikindustrie herrschende Ästhetik der populären Musik verinnerlicht und gelernt, sie im Rahmen der bestehenden Produktionsverhältnisse umzusetzen. Deshalb war es ihm ohne Umstände möglich, innerhalb von zweieinhalb Jahren sieben Alben und zehn Singles (inkl. der ersten Blue System-Produktionen) zu komponieren, zu produzieren, zu bewerben und mit ihnen auf Tour zu gehen.

Diese ungeheure Produktivität ist nur zu bewerkstelligen, wenn ein hohes Maß an Arbeitsteiligkeit herrscht. Und hier kommt ein anderer, bisher

14 »Ich setzte mich, wie ich das immer so mache, in mein Studio, schaltete das Keyboard ein. Klimperte ein bisschen auf den Tasten rum, sang ein paar Textideen dazu und ließ dabei ein Band mitlaufen. Das machte ich Stunde um Stunde. Komponieren ist für mich ein bisschen wie Mathe: Ich mache was dazu und drei im Sinn und einen lass [sic] ich fallen, bis zum Schluss der Song steht « (Bohlen 2002: 182). Nach einer kommerziell nicht erfolgreichen Produktion mit der von ihm verehrten Dionne Warwick zieht er die Lehre: »Nie wieder ein Song für die Kritikerloge. Ab jetzt nur noch für die Ränge und das Parkett « (ebd.: 191). 
nicht genannter Faktor der Musik von Modern Talking ins Spiel, nämlich das Studio-Team, bestehend aus dem Produzenten Luis Rodriguez, dem Musiker und Toningenieur Ralf Stemmann sowie den Sängern Rolf Köhler, Detlef Wiedeke, Michael Scholz und Birger Corleis. Diese Musiker waren für die Aufnahmen aller Modern Talking-Alben zuständig. ${ }^{15}$ Arbeitsteiligkeit, so lehrt es die Ökonomie, setzt wiederum ein hohes Maß an Kommunikation und Standardisierung voraus. Da Bohlen durch die erwähnten Aktivitäten oft die Teilnahme an der Produktion der Musik nicht möglich war, konnte er nur das fertige Produkt abnehmen. Und dieses wurde, um zu einer möglichst schnellen und reibungslosen Produktion beizutragen, an den einmal etablierten Modern Talking-Standards orientiert. Denn als erfahrener Angehöriger der Musikindustrie ahnte Bohlen (und sicher auch sein Label), dass der mit »You're My Heart, You're My Soul « relativ unerwartet hereingebrochene Erfolg nicht von langer Dauer sein würde. ${ }^{16}$ Insofern ist in der Standardisierung ihrer Musik ein Ergebnis des arbeitsökonomisch bedingten Versuchs zu sehen, die Marktfähigkeit der Musik möglichst rasch zu kapitalisieren.

Nicht unberücksichtigt bleiben darf dabei die Label-Umgebung, denn die derart entstandene Musik musste schnell in mediale Knotenpunkte und schließlich in die Geschäfte zum Verkauf gebracht werden. Dazu benötigte man erfahrene Verkäufer populärer Musik, die über die notwendigen Verfahren, Ansprechpartner und das ökonomische Kapital verfügten. Die HansaMusikproduktion ist ein für den bundesdeutschen Raum wichtiger Player auf dem Markt der populären Musik. Sie wurde 1964 von Thomas Meisel zusammen mit seinem Bruder Peter sowie dem Schlagerkomponisten Christian Bruhn nach Vorbild der US-amerikanischen Independent-Labels gegründet; die Meisels sind die Kinder des Operetten-Sängers Will Meisel, der die Meisel-Musikverlage gründete, die heute 36 Unterverlage und 16 eigene Labels umfassen. Bruhn schied nach kurzer Zeit auf eigenen Wunsch hin aus der

15 Dies bestätigt Frank Farian in seinem literarisch anspruchslosen, indes machtpolitisch hochgradig ambitionierten Versuch der Ausschaltung seines Konkurrenten. Er nennt dieselben Sänger und bezeichnet Luis Rodriguez als federführend im Studio, die von Bohlen hereingereichten Demos umzusetzen (Farian/Kaltwasser/Rudorf 2004: 55, 68ff. u. 147). Christian Bruhn spricht von »Bohlens Fabrikarbeitern« (Bruhn 2005: 393).

16 Zum Zeitpunkt der Veröffentlichung der ersten Single gab es zwar die Interpreten Modern Talking, aber keine Band, geschweige denn eine Marktstrategie. Thomas Anders wollte, das war die Vorbedingung für die Produktion, unter keinen Umständen mit "You're My Heart, You're My Soul in Verbindung gebracht werden, weil er negative Auswirkungen des englischsprachigen Songs auf seine angestrebte Schlagersängerkarriere befürchtete. Hans Blume, damaliger Geschäftsführer der Hansa, stellt die Abläufe in der Industrie richtig: »Überhaupt fangen die Pop-Karrieren häufig mit einem guten Titel an, und die Interpreten werden erst nachträglich gesucht « (zit. n. Eidam/Schröder 2001: 229). 
Hansa-Musikproduktion aus; in seiner Autobiografie bezeichnet er, in fünfter Ehe lebend, diese Trennung als »wohl größten Fehler meines Lebens « (Bruhn 2005: 136f.). Viele MusikerInnen des deutschen Schlagers waren bei der Hansa als Interpreten - z.B. auch Thomas Anders vor Modern Talking und/oder Komponisten - bspw. Gunter Gabriel, der u.a. den oft vergessenen Song »Ich laß dir einen Kochtopf, laß du mir mein Bier « für Peter Alexander (1979) schrieb - unter Vertrag, aber auch Boney M. $^{17}$ Der Vertrieb lief zunächst über Ariola und wurde 1985 dann an Bertelsmann verkauft, ab 1987 war Hansa dann bis auf die Hansa-Studios Teil des Konzerns. Nach einigen Jahren bei BMG und SonyBMG ist das Hansa-Label heute bei Sony Music Entertainment. Übrigens: Bertelsmann ist Mehrheitsaktionär der RTL Group, eine Bertelsmann-Tochterfirma produziert Deutschland sucht den Superstar und Dieter Bohlen ist noch immer bei Hansa unter Vertrag - folglich auch seine Produktionen etwa der DSDS-Reihe. Diese personellen wie strukturellen Verflechtungen der jüngeren deutschen Musik- und Unterhaltungsgeschichte nehmen ihren Ausgang also in Bohlens Tätigkeit und seinen ersten Erfolgen mit Modern Talking für die Hansa; seine heutige Medien- und Marktmacht liegen hier begründet. ${ }^{18}$

Zum zweiten aber, und hier greife ich zurück auf die obige Prämisse der Subjektbezogenheit der Analyse, sind diese Ergebnisse und ihre Darstellung die Produkte meiner Auseinandersetzung mit Modern Talkings Musik. Doch was höre bzw. was verstehe ich eigentlich? Und warum? Ich könnte es mir einfach machen und behaupten, dass die festgestellte Standardisierung weniger musikalischer Mittel meine Abneigung der Musik begründen könnte. Jedoch: Meine eigentümliche Faszination für diese Musik und die Tatsache, dass ich im Jahr 2013 mit Ralf von Appen und Markus Frei-Hauenschild an einer 321 Songs umfassenden Korpus-Analyse aller Rolling Stones-Songs gearbeitet habe (vorgeblich ebenso simple Musik, deren Analyse mir aber wesentlich mehr Freude bereitete) ${ }^{19}$ müssten dann entweder verschwiegen

17 Frank Farian gründete sogar mit der Edition Intro Meisel den Co-Verlag FAR Musikverlag $\mathrm{GmbH}$, wo als erster Millionenhit »Ma Baker « verlegt wurde.

18 Die langjährige persönliche Bekanntschaft mit heute mächtigen Angehörigen der Industrie ist dabei sicherlich hilfreich. Stellvertretend für viele andere ist hier bspw. André (Andi) Selleneit zu nennen, ein alter Wegbegleiter von Dieter Bohlen, der als Mitarbeiter der Hansa mit Bohlen vor Modern Talking das Projekt Steve Benson gegründet hatte. Selleneit folgte Hans Blume ab 1992 als Geschäftsführer der Hansa, ab 1997 war er Geschäftsführer der BMG Berlin, 2005 gründete er die DEAG (Deutsche Entertainment AG), ein $360 \mathrm{Grad}$-Label, bei dem u.a. David Garrett unter Vertrag ist, ab 2010 war er bei Sony und ist heute wieder bei der DEAG.

19 Die Ergebnisse wurden 2013 auf der PopMAC-Konferenz in Liverpool und der GfPM-Tagung in Gießen, 2014 auf der EuroMAC in Leuven vorgestellt. 
oder aber, und das soll hier passieren, zum Gegenstand gemacht werden. Meine analytische Rezeption - die Analyse ist ein besonderer Fall der Rezeption - unterliegt kulturellen, beruflichen und sozialen Kontexten, die weder immer bewusst gemacht noch ausgeschaltet, aber in der »radikalen Selbstreflexivität « (Doehring 2012: 37f.) als Teil des analytischen Prozesses herausgearbeitet werden können. Und nur mit ihrer Hilfe wird meine ambivalente Rezeption der Musik verständlich, wie sie eingangs dieses Beitrags beschrieben wurde.

Als in den 1980er Jahren in der BRD Aufgewachsener ist mir Modern Talking bereits lange vor der beruflichen Auseinandersetzung mit ihrer Musik geläufig. Ich erinnere mich an ihre Videos, die in der TV-Sendung Formel Eins gezeigt wurden, Fernsehauftritte bei Wetten daß...?, Artikel in der Bravo sowie natürlich an ihre Musik, die ich aus dem Radio auf Kassette aufgenommen habe und die in der Tanzschule, der Dorfdisco oder auf der Kirmes am Autoscooter lief (und wenn sich die Blaskapelle progressiv gab, auch im Festzelt). Dies ist mein Blick, der sich erheblich von Rezeptionen im Iran oder bspw. auch Russland unterscheidet: ${ }^{20}$ Dort war - und ist - die Musik ein Inbegriff westlicher Popmusik, hedonistisch und damit hochgradig politisch. Sie war und ist Ausdruck von Freiheit - wohingegen ich mich an eine kurze erste Begeisterung, mit dem Heranwachsen aber dann an den bundesrepublikanischen Mief der ersten Kohl-Jahre und per Discofox zwangsregulierte Bewegung erinnere.

Ähnlich spezifisch gestaltet auch der berufliche Kontext meine Rezeption. Modern Talking ist, wie dargestellt, ein fremdes, möglicherweise illegitimes Objekt für meine Heimatdisziplin. Überlegungen bezüglich möglicher Auswirkungen meiner Beschäftigung mit Modern Talking - Prozesse der Stigmatisierung, gar Ausgrenzung - begleiten mein Vorgehen, das nun umso mehr bemüht ist, den diskursiven Regeln der Disziplin entsprechend zu handeln und das heißt: den Gegenstand zu formen. Wie sonst wenn nicht durch die analytische Methode sollte eine Legitimierung von Gegenstand und Autor erfolgen?

Des Weiteren beeinflussen meinen Blick auf die Musik Modern Talkings ein habituell erworbenes Musikkonzept sowie ein Gender-Aspekt. Der anfänglich noch präpubertären Freude an der Musik Modern Talkings, die ich mit meinen Schulfreunden teilte, folgte rasch die bis heute anhaltende Abneigung, die sich ausbildete, als ich älter wurde und begann, in ersten

20 Die Sowjetunion war bereits 1978 Tourneeziel einer bundesdeutschen HansaProduktion: Boney M. traten dort für zehn Konzerte auf. Ab 1986 durften Modern Talking-Alben in der Sowjetunion verkauft werden, 1987 spielte das Duo dann eine erste von vielen folgenden Tourneen dort. 
Rock- und Blues-Bands sowie Orchestern zu spielen. Nun machte ich >rich-

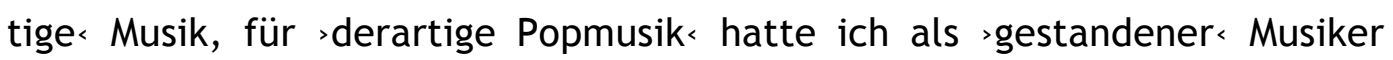
nichts mehr übrig (zu haben). Dieser Wandel ist auch auf durch das von Thomas Anders repräsentierte Männlichkeitsbild zurückzuführen, das die männliche Norm der Mittachtziger Jahre infrage stellte - eine Norm, die zu jener Zeit der pubertierenden Orientierungsnot sicherlich auch für einige Jahre die meine war. Repräsentierte Männlichkeit bestimmt auch die heutige professionelle Beschäftigung mit Modern Talking, allerdings unter umgekehrten Vorzeichen: Es gelingt mir nicht, die mir machtvoll über die Konzerne Springer und Bertelsmann übermittelte media persona Dieter Bohlen ${ }^{21}$ und die durch sie vertretene Ideologie einer neoliberalen Leistungs- bzw. Verdrängungsgesellschaft sowie ihre Verkörperung eines von mir als unhaltbar befundenen Machismo auszublenden: »Talent + arbeiten + arbeiten + arbeiten + arbeiten - dann kommt irgendwann auch die Kohle. Und: Haste Kohle, haste Frauen« (Bohlen 2002: 9).

Nichts davon kann und darf vergessen werden, denn diese kulturellen, professionellen und sozialen Aspekte beeinflussen jeden Aspekt meiner analytischen Arbeit. Ich höre etwa sehr deutsches bzw. falsches Englisch (bspw. in »Just We Two (Mona Lisa)«, auf Modern Talking 1986c), ich höre das Bemühen um (in meinen Ohren nie erreichten) Anschluss an internationale Popmusik, bspw. in den Bee Gees-artigen Post-Chorus-Chören. Ich vermisse innerhalb dieser sehr vorhersagbaren musikalischen Anlage die (mikro-)rhythmische Bewegung, wie ich sie in afroamerikanischer Tanzmusik der 1980er Jahre höre. Und ich kann nirgendwo eine flamboyante Campiness entdecken, wie ich sie in britischer Tanzmusik jener Zeit finde - unzweifelhaft war es Bohlen und Anders um anderes gegangen, als die dominanten Genderregeln spielerisch infrage zu stellen:

»Modern Talking war tot. Nach den bonbonfarbenen Fallschirmseiden-Albträumen, dem Lipgloss und dem ganzen hoch, höher, am höchsten Gequieke wollte ich endlich wieder als richtiger Mann auf der Bühne stehen« (Bohlen 2002: 124).

21 Nur ein Beispiel: Bohlens Autobiografie Nichts als die Wahrheit wurde mit Dr. med. dent. Katja Kessler verfasst, Klatsch-Kolumnistin bei der BILD-Zeitung, seit 2002 mit dem Gesamtherausgeber der BILD-Gruppe im Axel Springer-Verlag Kai Diekmann (»Schatzi«, s.u.) verheiratet. Kessler veröffentlichte außerdem Bücher wie Frag mich Schatzi (2009), Das Schatzi-Experiment (2011) oder Silicon Wahnsinn. Wie ich mal mit Schatzi nach Kalifornien auswanderte (2014). Nichts als die Wahrheit erschien im Heyne-Verlag, der zu dieser Zeit zur Axel Springer-Gruppe gehörte und heute bei Random House ist, der Dachgesellschaft aller Bertelsmann-Verlage. 
»Ich habe ganz sicherlich nichts gegen Homosexuelle, es gibt einige in meinem Freundeskreis. Liebenswerte und tolle Menschen, aber wenn man mich als schwul verkaufen will, und dann auch noch aus reinen Profitgründen, möchte ich wenigstens vorher von den Plattenbossen gefragt werden« (Anders 2011: 158f.).

Höre ich Modern Talking, höre ich deutsche Musiker aus der Welt des Schlagers, die sich den Regeln und Ansprüchen des Musikmarkts fügen, um mehr Geld - und bohlenscher Logik zufolge: mehr Frauen - zu erbeuten. Ein kurzes Fallbeispiel mag dies verdeutlichen: Der Song »You And Me« (auf Modern Talking 1987b) ist deutlich durch den U2-Song »I Still Haven't Found (What I'm Looking For)« (1987) inspiriert. Die im Modern Talking-Intro erklingende Lead-Gitarre spielt sogar auf die Gesangslinie der Zeilen »Only to be with you « aus dem U2-Song an. Beide Songs bedienen sich einer Dur-Pentatonik, die harmonische Anlage der Gesang-Parts beginnt identisch mit einer achttaktigen Struktur aus I / I / I / I / IV / IV / I / I. Aber danach gehen die Songs getrennte Wege. Im U2-Song werden diese acht Takte wiederholt, es folgt dann die Refrain-Zeile über eine wiederholte V / IV / I / I-Sequenz und damit wird klar, dass es sich um eine gedehnte Blues-Form handelt. In »You And Me « dagegen läutet in Takt 8 auf die dritte und vierte Zählzeit die Kadenz IV - V die Wiederholung ein, die am Ende in den Pre-Chorus (vier Takte V) übergeht, der den einteiligen Chorus (IV / IV / I / I / IV / IV / V / V) vorbereitet. Als Hörer und Musiker bluesidiomatischer Musik kann ich nicht umhin, die Fortführung dieser Blues implizierenden ersten acht Takte als >falsch wahrzunehmen, als in der BRD sozialisierter Hörer erkenne ich sie zudem als Schlager, gerade aufgrund der Schlager-typischen Kadenz in Takt 8. Mein Urteil wird außerdem bestärkt durch die Dreiklangs-Melodik der ersten vier Takte, gesungen von Anders' Stimme, die mir mit ihrem Sound, der klaren Intonation und der mehrfachen Dopplung durch andere Stimmen Schlager signalisiert (wohingegen Bonos raue Stimmgebung, Intonation und Phrasierung diese Zuschreibung unmöglich machen).

Es soll hier nicht darum gehen, Bohlens Arbeitsweise infrage zu stellen - die Inspiration durch andere Titel ist in der Musikindustrie lange und weit verbreitet und, wie gezeigt, durch die Produktionsumstände bedingt. ${ }^{22}$ Sondern ich will klarstellen, dass die klangliche Struktur der Musik für die Rezeption bedeutsam ist und die Analyse mit der anschließenden Interpretation anschlussfähige Ergebnisse liefern kann, die zwar hochgradig sub-

22 Übrigens: »I Still Haven't Found What I'm Looking For « ist als Albumtrack drei Monate, als Single einen Monat vor Romantic Warriors veröffentlicht worden. Bohlen musste und konnte innerhalb der beschriebenen Strukturen schnell arbeiten. 
jektiv sind, aber nie ahistorisch oder kulturell unverbunden. Eine bspw. iranische Musikwissenschaftlerin wird auf Basis dieser Daten zu anderen Interpretationen gelangen, sollte sie die dort stärker bewachten Grenzen der Disziplin überschreiten. Ausgestattet mit dem notwendigen, nicht bloß wie in meinem Falle durch gelegentliche Familienbesuche erworbenen, kulturellen Wissen über den Iran könnte sie zeigen, inwiefern dieselben Klänge eine andere Rezeption ermöglichen.

\section{Ausblick}

Was kann ich also als deutscher Musikwissenschaftler mithilfe der Analyse an einem populären und zugleich fremden Repertoire verstehen? Ich verstehe und zeige, dass die klangliche Struktur in ihrer Einheitlichkeit erstens sozialgeschichtlich relevante Erkenntnisse liefert und dass ich zweitens mit dieser Musik wenig anfangen kann - etwas, was ich mindestens mit meinen im bundesdeutschen Kulturraum sozialisierten Freunden und Kollegen teile, die ich zu Beginn zitiert habe. Jedoch produziert eine spezifizierende Methode spezifisches Wissen, in diesem Fall über ein hoffentlich verständlicheres Unverständnis der Musik von Modern Talking. Nicht erklären kann meine Korpus-Analyse, was die Fans von Modern Talking verstehen, die seit damals am Ball geblieben sind, - oder eben die Freude der Hörer im Iran an dieser Musik, die sie unter anderen kulturellen und politischen Umständen rezipieren.

Der subjective turn der Analyse ist eine methodologisch wichtige Entwicklung. Doch wird es in der künftigen analytischen Arbeit darum gehen müssen, auch das Musikverstehen Dritter zu erfassen und per Analyse an die klangliche Struktur zu binden. Die hier vorgelegten Daten der KorpusAnalyse mögen dafür als Grundlage dienen, doch sind sie an der musikalischen Praxis anderer Menschen und Kulturen zu überprüfen. Die Methode der Gruppen-Analyse (Appen/Doehring/Helms/Moore 2015) scheint mir dafür hochgradig geeignet, ergänzt durch ethnografische Methoden wie Interviews und teilnehmende Beobachtungen bei der Rezeption von Musik, ob im iranischen Auto oder bei Konzerten, Fantreffen und den anschließenden Partys samt Tanz zu Modern Talkings Musik. Denn dies alles sind gültige Verständnisse populärer Musik, die die hier von mir als erstes Verstehensangebot vorgelegte Interpretation sicher bereichern und in Teilen falsifizieren werden. 


\section{Literatur}

Adorno, Theodor W. (1996). Einleitung in die Musiksoziologie. Zwölf theoretische Vorlesungen. Frankfurt/M.: Suhrkamp.

Anders, Thomas, mit Tanja May (2011). 100 Prozent anders. Mein Leben - und die Wahrheit über Modern Talking, Nora und Dieter Bohlen. Höfen: Edition Koch.

Appen, Ralf von / Doehring, André (2014). »Analyse populärer Musik. Madonnas >Hung Up .«In: Populäre Musik. Geschichte - Kontexte - Forschungsperspektiven. Hg. v. Ralf von Appen, Nils Grosch und Martin Pfleiderer (= Kompendien Musik 14). Laaber: Laaber, S. 219-240.

Appen, Ralf von / Doehring, André / Helms, Dietrich / Moore, Allan F. (2015). »Introduction. «In: Song Interpretation in 21st-Century Pop Music. Hg. v. dens. Farnham: Ashgate, S. 1-6.

Appen, Ralf von / Frei-Hauenschild, Markus (2015). »AABA, Refrain, Chorus, Bridge, Pre-Chorus - Song Forms and their Historical Development.« In: Samples 13; www.gfpm-samples.de/Samples13/appenfrei.pdf (Zugriff 10.3.2015).

Blaukopf, Kurt (1984). Musik im Wandel der Gesellschaft. Grundzüge der Musiksoziologie. München, Kassel: dtv / Bärenreiter.

Bohlen, Dieter, mit Katja Kessler (2002). Nichts als die Wahrheit. München: Heyne.

Bruhn, Christian (2005). Marmor, Stein und Liebeskummer. Meine Welt ist die Musik. Erinnerungen, Gedanken und Gefühle von ihm selbst aufgeschrieben. Berlin: Schwarzkopf \& Schwarzkopf.

Byron, Tim (2014). »An inside look at how Pandora can pick the next song you want to hear.«In: the VINE vom 25 September; http://thevine.com.au/music/news/ an-inside-look-at-how-pandora-can-pick-the-next-song-you-want-to-hear-201409 25-287192 (Zugriff 25.9.2014).

Calella, Michele / Urbanek, Nikolaus (Hg.) (2013). Historische Musikwissenschaft. Grundlagen und Perspektiven. Stuttgart, Weimar: Metzler.

Doehring, André (2011). Musikkommunikatoren. Berufsrollen, Organisationsstrukturen und Handlungsspielräume im Popmusikjournalismus (= texte zur populären musik 7). Bielefeld: transcript.

Doehring, André (2012). »Probleme, Aufgaben und Ziele der Analyse populärer Musik. « In: Black Box Pop. Analysen populärer Musik. Hg. v. Dietrich Helms und Thomas Phleps (= Beiträge zur Popularmusikforschung 38 ). Bielefeld: transcript, S. 23-42.

Doehring, André (2015). »Andrés's >New For U< - New For Us. On Analysing Electronic Dance Music. In: Song Interpretation in 21st-Century Pop Music. Hg. v. Ralf von Appen, André Doehring, Dietrich Helms und Allan F. Moore. Farnham: Ashgate, S. 133-155.

Eidam, Klaus / Schröder, Rudolf (2001). Die Hit-Fabrik. Zweiter Teil der Geschichte eines Berliner Musikverlages. Berlin: Edition Intro Meisel.

Farian, Frank / Kaltwasser, Dieter / Rudorf, Reginald (2004). Stupid dieser Bohlen. Die Wahrheit und nichts als die Wahrheit über den Pop-Hochstapler. Friedrichsdorf: Frank's Kleiner Buchverlag.

Jost, Ekkehard (1999a). »Über einige Probleme der jazzmusikalischen Analyse.« In: Jazzforschung/Jazz Research 31, S. 11-18.

Jost, Ekkehard (1999b). »Zum Sprachcharakter von Musik im allgemeinen und Jazz im Speziellen. «In: Jazz und Sprache. Sprache und Jazz. Hg. v. Wolfram Knauer (= Darmstädter Beiträge zur Jazzforschung 5). Hofheim: Wolke, S. 87-106. 
La Motte-Haber, Helga de (1982). »Umfang, Methode und Ziel der Systematischen Musikwissenschaft." In: Systematische Musikwissenschaft. Hg. v. Carl Dahlhaus und Helga de la Motte-Haber (= Neues Handbuch der Musikwissenschaft 10). Wiesbaden, Laaber: Akademische Verlagsgesellschaft Athenaion, Laaber, S. 1-24.

Moore, Allan F. (2009). »Interpretation: So What?« In: The Ashgate Research Companion to Popular Musicology. Hg. v. Derek B. Scott. Farnham: Ashgate, S. 411425.

Moore, Allan F. (2012). Song Means. Analysing and Interpreting Recorded Popular Song. Farnham: Ashgate.

Phleps, Thomas (2014). »Freunde bis ans Ende der Zeit - Schlagertümlichkeit reloaded."In: Rollenspiele. Musikpädagogik zwischen Bühne, Popkultur und Wissenschaft. Festschrift für Mechthild von Schönebeck zum 65. Geburtstag. Hg. v. Thomas Erlach und Burkhard Sauerwald. Frankfurt/M.: Peter Lang, S. 433-449.

Pfleiderer, Martin / Grosch, Nils / Appen, Ralf von (2014). "Populäre Musik und Wissenschaft. Forschungstraditionen und Forschungsansätze." In: Populäre Musik. Geschichte - Kontexte - Forschungsperspektiven. Hg. v. Ralf von Appen, Nils Grosch u. Martin Pfleiderer (= Kompendien Musik 14). Laaber: Laaber, S. 200-209.

Rösing, Helmut (2002). »>Popularmusikforschung in Deutschland - von den Anfängen bis zu den 1990er Jahren.« In: Musikwissenschaft und populäre Musik. Versuch einer Bestandsaufnahme. Hg. v. Helmut Rösing, Albrecht Schneider und Martin Pfleiderer (= Hamburger Jahrbuch für Musikwissenschaft 19). Frankfurt/M.: Peter Lang, S. 13-35.

Tagg, Philip (1992). »Towards a Sign Typology of Music."In: Secondo Convegno Europeo Di Analisi Musicale. Hg. v. Rosanna Dalmonte und Mario Baroni. Trient: Università degli Studi di Trento, S. 369-378; http://tagg.org/articles/trento91. html (Zugriff 22.7.2015).

Wald, Elijah (2014). »Forbidden Sounds: Exploring the Silences of Music History.« In: Geschichte wird gemacht. Zur Historiographie populärer Musik. Hg. v. Dietrich Helms und Thomas Phleps (= Beiträge zur Popularmusikforschung 40). Bielefeld: transcript, S. 25-39.

\section{Diskographie}

Alexander, Peter (1979). »Ich laß dir den Kochtopf, laß du mir mein Bier.« Auf: Ein Abend mit Peter Alexander. Die neuen Schlagererfolge. Ariola 200798.

Blue System (1987a). »Sorry Little Sarah. « Hansa 609469.

Blue System (1987b). Walking On A Rainbow. Hansa 208696.

Modern Talking (1984). »You're My Heart, You're My Soul.« Hansa 601496.

Modern Talking (1985a). »You Can Win If You Want. « Hansa 601670.

Modern Talking (1985b). The 1st Album. Hansa 206818.

Modern Talking (1985c). »Cheri, Cheri Lady.«Hansa 601950.

Modern Talking (1985d). Let's Talk About Love. The 2nd Album. Hansa 610522.

Modern Talking (1986a). »Brother Louie.« Hansa 602157.

Modern Talking (1986b). »Atlantis Is Calling (S.O.S. For Love). « Hansa 608239.

Modern Talking (1986c). Ready For Romance. The 3rd Album. Hansa 257705.

Modern Talking (1986d). »Geronimo's Cadillac.« Hansa 608620. 
Modern Talking (1986e). In The Middle Of Nowhere. The 4th Album. Hansa 258039. Modern Talking (1986f). »Give Me Peace On Earth. « Hansa 608778.

Modern Talking (1987a). »Jet Airliner.« Hansa 609138.

Modern Talking (1987b). Romantic Warriors. The 5th Album. Hansa 258400.

Modern Talking (1987c). »In 100 Years. " Hansa 609543.

Modern Talking (1987d). In The Garden Of Venus. The 6th Album. Hansa 258770.

Modern Talking (1998). Back For Good. The 7th Album. Hansa 74321 57358-2.

U2 (1987). »I Still Haven't Found What I'm Looking For.« Auf: The Joshua Tree, Island 258219.

\begin{abstract}
The music of German pop duo Modern Talking has been widely neglected in the field of popular music studies and musicology as well. I present the results of a corpus analysis of the six albums Modern Talking published between 1985-87 in which I show a high degree of similarity concerning tempo, formal and harmonic layout, rhythm and instrumentation. My interpretation claims these musical features to result in the circumstances of producing popular music in Germany in the mid-1980s. Furthermore, methodological reflections on the subjective turn of analysis show how my interpretation is influenced by cultural, professional, and social aspects. Hence, I call for further analytical research on popular electronic dance music - especially since the reception of their music in other parts of the world as e.g. in Iran differs significantly from my own.
\end{abstract}

